\title{
Calibration accuracy of laser calorimetry for common crystal geometries
}

Yannick Willer, Liu Hao, Istvan Balasa, Detlev Ristau

Yannick Willer, Liu Hao, Istvan Balasa, Detlev Ristau, "Calibration accuracy of laser calorimetry for common crystal geometries," Proc. SPIE 10447, Laser-Induced Damage in Optical Materials 2017, 104471V (29 November 2017); doi: $10.1117 / 12.2281335$

SPIE. Event: SPIE Laser Damage, 2017, Boulder, Colorado, United States 


\title{
Calibration accuracy of laser calorimetry for common crystal geometries
}

\author{
Yannick Willer $^{\mathrm{a}}$, Liu Hao ${ }^{\mathrm{b}}$, Istvan Balasa ${ }^{\mathrm{ia}}$, Detlev Ristau ${ }^{\mathrm{a}}$ \\ ${ }^{a}$ Laser Zentrum e.V., Laser Components Department, Characterization Group, Hollerithallee 8, \\ 30419 Hannover, Germany \\ ${ }^{b}$ Leibniz Universität Hannover, Laboratory of Nano and Quantum Engineering, Schneiderberg 39, \\ 30167 Hannover, Germany
}

\begin{abstract}
An established method for precise determination of optical absorption is the so called laser calorimetry. According to ISO $11551^{1}$ laser calorimetry is preferred to other photothermal test methods, because of its capability to deliver absolute calibration. Many optical materials have low heat conductivity, which can affect the calibration significantly. The timeand spatial dependent temperature profile in a sample of materials with low heat conductivity requires accurate temperature measurement strategies to determine material-independent and absolutely calibrated absorption values. For thin cylindrical samples, ISO 11551 provides a strategy to compensate heat conductivity effects. The optimal temperature sensor position, where accordingly calibrated measurement results ${ }^{2}$ can be obtained, is simply based on the symmetric sample geometry. For thick geometries an additional temperature distribution along propagation direction of the heating beam must be considered. The current version of ISO 11551 does not provide a sophisticated solution for this problem, because the heating scheme of a sample is usually unknown. Therefore, a reliable calibration procedure can only be applied to samples of well-known absorption properties of surfaces and bulk material. Utilizing such kind of specifically prepared reference samples in combination with Finite Element Method (FEM) calculations, a general measurement and data evaluation concept based on laser calorimetry is presented, that allows deriving absolutely calibrated absorption measurement results for rectangular sample geometries.
\end{abstract}

Keywords: laser calorimetry, ISO11551, absorption, finite heat conductivity, nonlinear optics, laser optics

\section{INTRODUCTION}

Since optical absorption changes the functionality of many laser components through multiple physical effects, such as thermal expansion and thermal lensing ${ }^{3}$, absorption measurements are of high importance. The measurement principle, which is mostly used for the determination of the absorption, is the so called laser calorimetry.

Other prominent measurement methods, for example photothermal surface-displacement techniques ${ }^{4-6}$, laser induced deflection $^{7,8}$, photothermal interferometry ${ }^{9,10}$ and thermal lensing techniques ${ }^{11,12}$, are very common and exhibit distinct advantages. They are much more suitable for spatially resolved absorption measurements ${ }^{13}$ than laser calorimetry, although they do not need physical contact with the sample, which can be important for certain measurements. A big problem with these techniques is the absolute calibration of the photothermal signal ${ }^{14}$, which requires exact knowledge of the optical and thermophysical parameters of the sample and it is rarely possible to gather all needed information ${ }^{15}$. Until now, only laser calorimetry is an established absolutely calibrated measurement procedure, according to the international standard. All evaluation methods of the laser calorimetry are based on a homogeneous temperature model (see Sec. 3), which is not useful for 'real' measurements, because the finite heat conductivity of 'real' optical materials is not considered. Under this condition, the evaluation of absorption data gained from laser calorimetry loses its simplicity, as the finite heat conductivity implies a temperature distribution which must be considered. Besides the heat conduction, other parameters, for example the sample geometry, material properties, heating as well as cooling time and the environmental conditions influence the evaluation of the absorption in real measurements.

\footnotetext{
${ }^{i}$ Istvan Balasa: E-mail: i.balasa@1zh.de, Telephone: +49 511 2788-476
} 
A viable detector position, at which the measurement result is nearly independent on the thermal diffusivity of the sample material, was found by Willamowski et al. ${ }^{16}$. ISO 11551 suggests a suitable detector position of thin cylindrical sample optics and the 'exponential method' (see Sec. 3) to evaluate the detected temperature curves. For samples with large thickness-surface-ratio, however, the additional temperature distribution along beam propagating direction must be considered, which is not included in ISO 11551 and is in general unknown. This work is based on the mentioned detailed studies ${ }^{16}$ with the aim to find suitable detector positions of common cuboid crystal geometries by calculation and experiments to verify absolutely calibrated absorption measurement results. Laser radiation at $193 \mathrm{~nm}$ is used to heat the samples.

\section{SAMPLE SETS AND THEIR PREPARATIONS}

In this work the influence of finite heat conductivity on the absorption measurements is studied. Therefore, the performance of multiple test samples with low heat conduction was compared to samples with considerable higher heat conductivity, to identify a suitable detector position. The test sample geometries were made of aluminum and ${ }^{\circledR} \mathrm{B} 270$ glass. Aluminum has a very high $\left(0,5 \mathrm{~cm}^{2} / \mathrm{s}\right)$ and ${ }^{\circledR} B 270$ a very low $\left(0,0042 \mathrm{~cm}^{2} / \mathrm{s}\right)$ thermal diffusivity, compared with other typical materials for absorption measurements (see Fig. 2.1). This high contrast in diffusivity is predestinated for finding a detector position at which heat conductivity effects can be neglected.

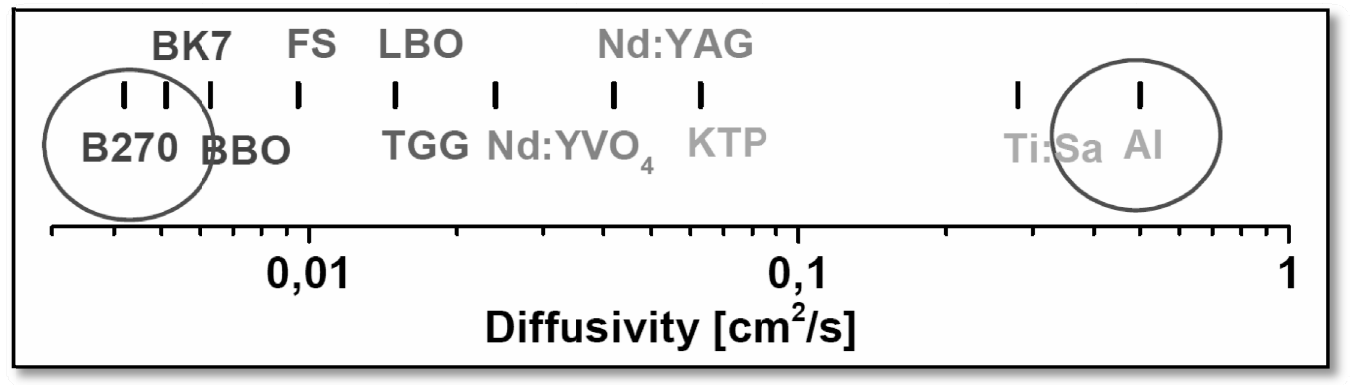

Fig. 2.1. Comparison of the diffusivity of many typical materials for absorption measurements. The test samples for this work were made of $\circledast \mathrm{B} 270\left(0,0042 \mathrm{~cm}^{2} / \mathrm{s}\right)$ and Aluminum $\left(0,5 \mathrm{~cm}^{2} / \mathrm{s}\right)$.

All acquired test samples are shown in Fig. 2.2, whereas viable apertures are $4 \times 4 \mathrm{~mm}^{2}, 10 \times 10 \mathrm{~mm}^{2}$ and $20 \times 20 \mathrm{~mm}^{2}$, and the respective thicknesses are $2 \mathrm{~mm}, 8 \mathrm{~mm}, 20 \mathrm{~mm}$, and $40 \mathrm{~mm}$. To compare the ${ }^{\circledR B} 270$ and aluminum samples (see left, middle in Fig.2.2), the absorption must be known in detail. This is attained by blackening the irradiation region of the front face with a graphite coating. However, even the absolute absorptance of this graphite coating is not known exactly, but it guarantees the same absorption for the aluminum and ${ }^{\circledR B} 270$ samples for identical heating power. The estimated absolute absorption is high and around $90 \%{ }^{2}$. To achieve detailed absolutely calibrated measurement results, wellprepared test samples with exactly known absorption are required. This could be achieved through 'modified' and blackened aluminum samples (see right in Fig. 2.2.), which have a known absolute absorption of $>99,5 \%$. All following experimental absorption measurement results are normalized to the 'modified' reference absorption results.

For the smallest aperture $\left(4 \times 4 \mathrm{~mm}^{2}\right)$, no modifications were made due to mechanical limitations, therefore, the resulting normalizations were averaged for all samples. Because of the highest thickness-surface-ratio, the $4 \times 4 \times 20 \mathrm{~mm}^{3}$ crystal is selected for comparison of the calculation and experimental absorption values. As a result of the blackened aluminum and ${ }^{\circledR B} 270$ samples, only the front face heating could be analyzed. In this work negative temperature coefficient thermistors (NTCs) were used as temperature sensors and were placed on the lateral surface with the possibility to vary the position along the laser beam propagation direction. The temperature sensor calibration was done electrically using a PT100 resistor. 


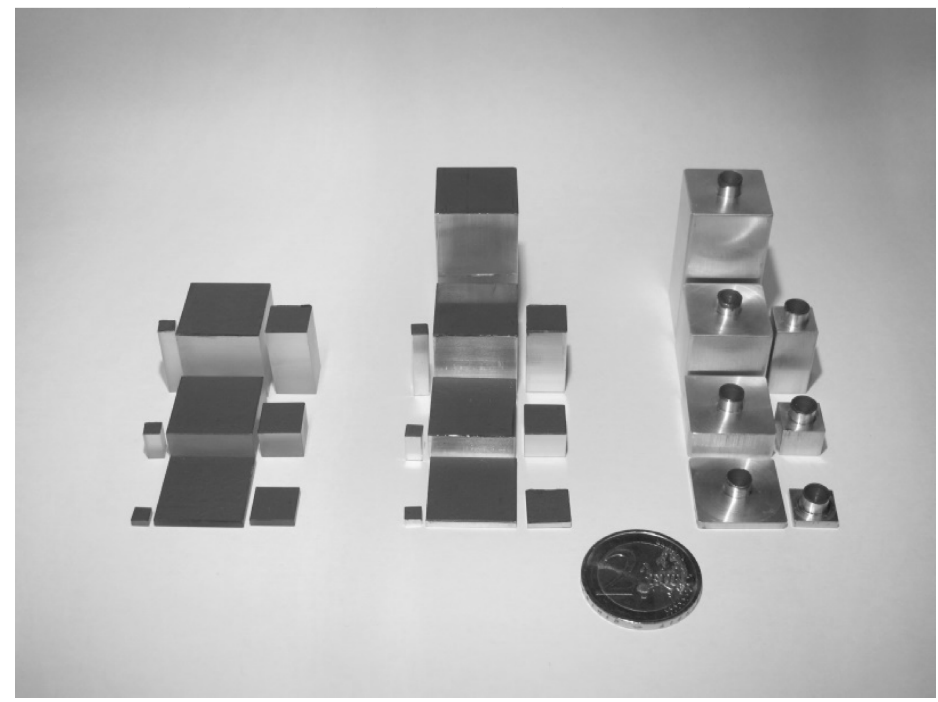

Fig. 2.2. All used test samples with the same graphite coating. Left: ${ }^{\circledR} B 270$ cuboid samples. Middle: aluminum cuboid samples. Right: With a cone 'modified' aluminum test samples. All viable apertures are $4 \times 4 \mathrm{~mm}^{2}, 10 \times 10 \mathrm{~mm}^{2} \mathrm{and}^{2}$ 20x20 $\mathrm{mm}^{2}$. The respective lengths are $2 \mathrm{~mm}, 8 \mathrm{~mm}, 20 \mathrm{~mm}$ and $40 \mathrm{~mm}$.

\section{UNDERLYING TEMPERATURE MODEL}

\section{Introduction of calculation}

It is reasonable to prove the existence of a suitable position on cuboid crystals by calculation before verifying it by experiments. The study of temperature distributions is accomplished with FEM using COMSOL Multiphysics ${ }^{17}$. FEM subdivides the crystal model into smaller domains called elements, over which a set of polynomial functions are solved approximately representing the heat transfer equation. COMSOL provides nine mesh sizes to subdivide the model automatically, from extremely coarse to extremely fine.

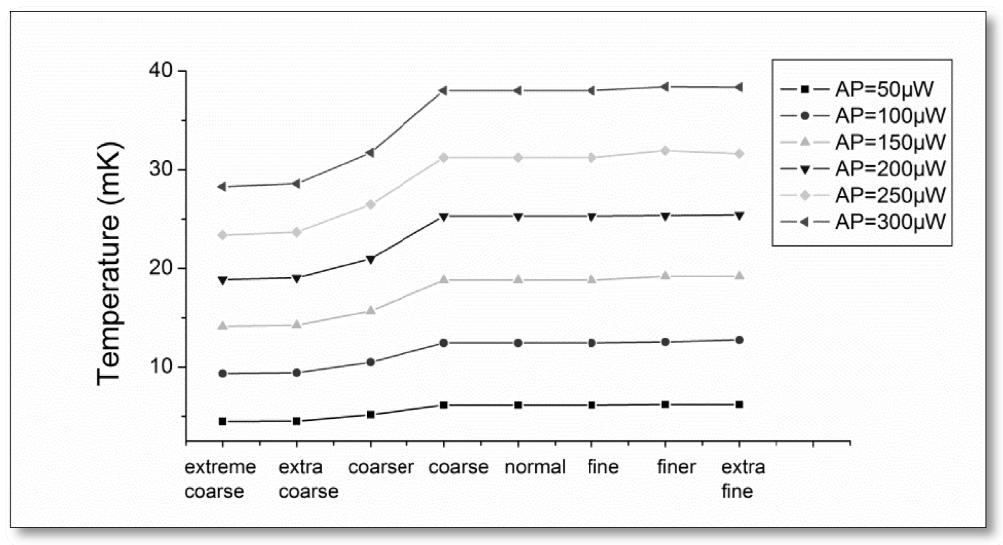

Fig. 3.1. Calculation comparison of different mesh sizes of $4 \times 4 \times 20 \mathrm{~mm}^{3}{ }^{\circledR} B K 7$ heated by certain source.

Different mesh sizes are tested by calculating the temperature of $4 \times 4 \times 20 \mathrm{~mm}^{3}{ }^{\circledR B K} 7$ heated by certain source. The $4 \times 4 \times 20 \mathrm{~mm}^{3}$ crystal is selected for calculation due to its highest thickness-surface-ratio. The sample is heated $120 \mathrm{~s}$ and then cooled $800 \mathrm{~s}$. The peak temperature results are compared in Fig. 3.1. Exceeding a certain mesh size, the calculation becomes independent of the mesh size. 'Fine' mesh is selected for following calculation. 
The temperature distribution is calculated by assuming certain absorption $A_{0}$. Both, 'exponential method' and 'pulse method' are normally used to evaluate the temperature-time curve, and the resulting absorption $A_{f i t}$ is compared with $A_{0}$. The evaluation is based on equation (eq. 1 ) $^{18}$, assuming a small temperature increase and a homogeneous temperature $T$ of sample and holder at all times.

$$
\frac{d T}{d t}=\frac{A P}{m c}-\gamma T
$$

In the equation $A$ represents absorption, $P$ laser power, $m$ sample mass, $c$ specific heat, $\gamma$ the heat loss coefficient, $t_{B}$ the irradiation time and $t$ time, respectively. Exponential evaluation fits the temperature curve in the heating and cooling period (equation 2), while pulse method only fits the cooling period (equation 3 ) and extrapolates to the time $\left(t_{1}+t_{2}\right) / 2$, as illustrated in Fig. 3.2.

$$
\begin{gathered}
\sum\left[T\left(t_{k}\right)-A_{h}-B_{h} \exp \left(-\gamma_{h} t_{k}\right)\right]^{2}=\min \\
A=\frac{A_{h} \gamma_{h} m c}{P} \\
\sum\left[T\left(t_{k}\right)-A_{c}-B_{c} \exp \left(-\gamma_{c} t_{k}\right)\right]^{2}=\min \\
A=\frac{T_{e x t} m c}{P t_{B}} 0.5 \gamma_{c} t_{B} / \sinh \left(\gamma_{c} t_{B} / 2\right)
\end{gathered}
$$

a)

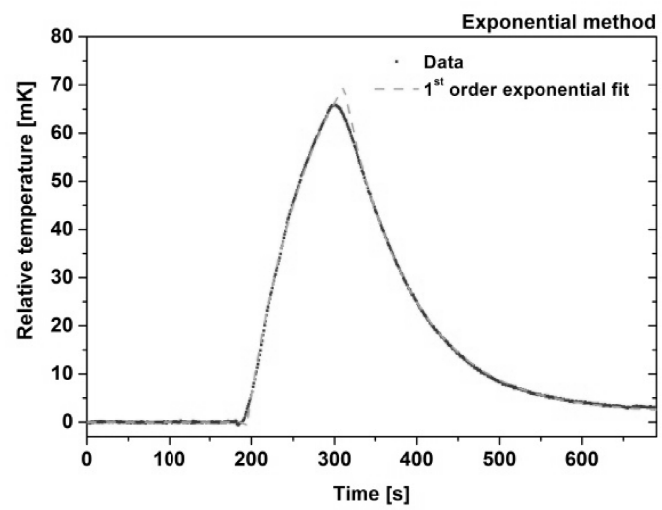

b)

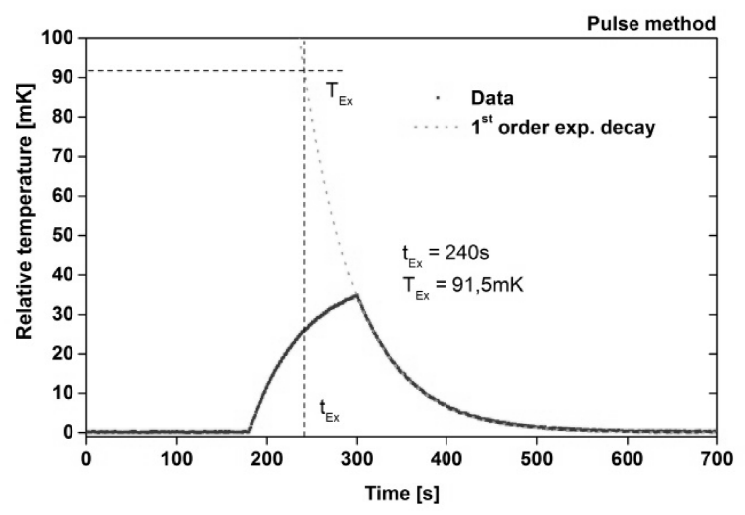

Fig. 3.2. Evaluation method scheme: (a) exponential method and (b) pulse method.

\section{Finite Element Method simulations}

To determine a suitable detector position, a set of samples with same geometry but varying thermal diffusivity is simulated. The temperature distributions at different sample positions are adopted along beam propagating direction $z$ and evaluated with exponential method with front surface heating scheme (Fig.3.3). Laser power $P$ and absorption $A_{0}$ are selected close to experimental calibration, that is $P=18 \mathrm{~mW}$ and $A_{0}=90 \%$. The evaluated absorption $A_{f i t}$ is divided by $A_{0}$ for comparison. As to Fig.3.3, the temperature decreases along $\mathrm{z}$ direction and for the indicated calibration factor close to 1 , the proper detector position is $z=8 \mathrm{~mm}$. However, the ratio $A_{f i t} / A_{0}$ of exponential method at $z=8 \mathrm{~mm}$ varies from 0.93 to 1.09 , leading to calibrating error as large as $9 \%$. 


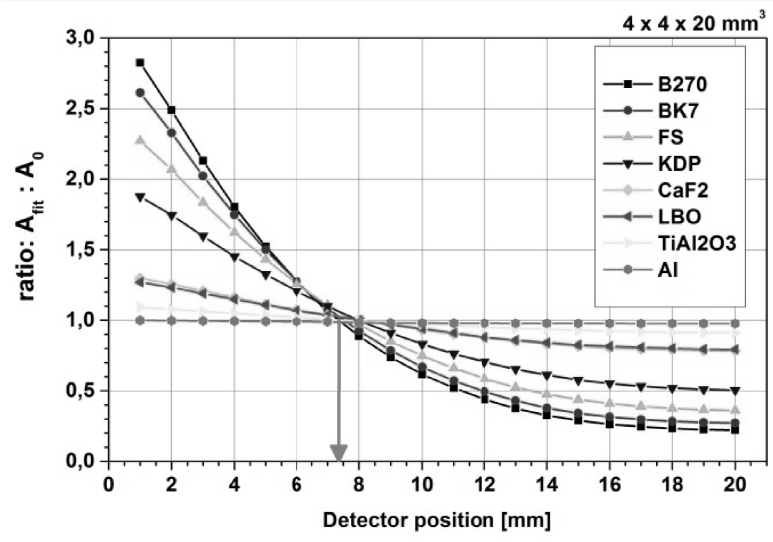

Fig.3.3. Simulated absorption of a suitable detector position for a $4 \times 4 \times 20 \mathrm{~mm}^{3}$ cuboid geometry with the exponential method for varying thermal diffusivities.

For a general overview two-face heating and bulk heating were simulated as well, although no experimental measurements for these heating schemes are available. In the case of two-face heating (Fig. 3.4.a), the suggested suitable detector position is $z=4.5 \mathrm{~mm}$ and $z=15.5 \mathrm{~mm}$. The calibration error at $z=4 \mathrm{~mm}$ or $z=16 \mathrm{~mm}$ is less than $5 \%$, and at $z=10 \mathrm{~mm}$ more than $10 \%$, respectively. As to bulk heating scheme (Fig. 3.4.b) the suitable detector position is nearly identical, while the temperature is much less dependent on the detector position. The calibration error at $z=4 \mathrm{~mm}$ or $z=16 \mathrm{~mm}$ is less than $2 \%$ while at $z=10 \mathrm{~mm}$ it is about $5 \%$.

a)

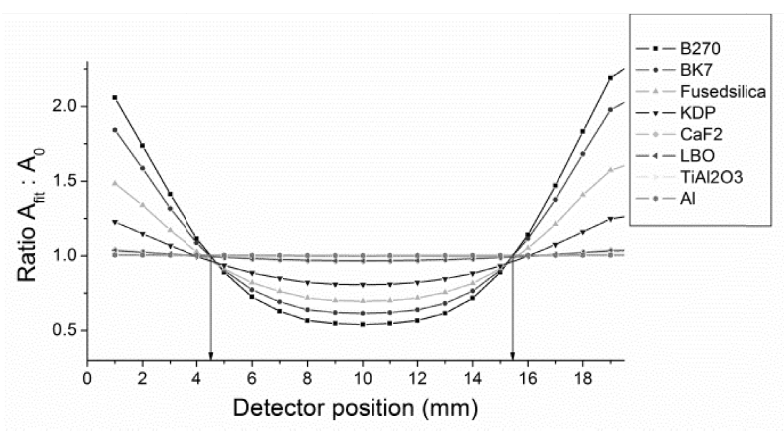

b)

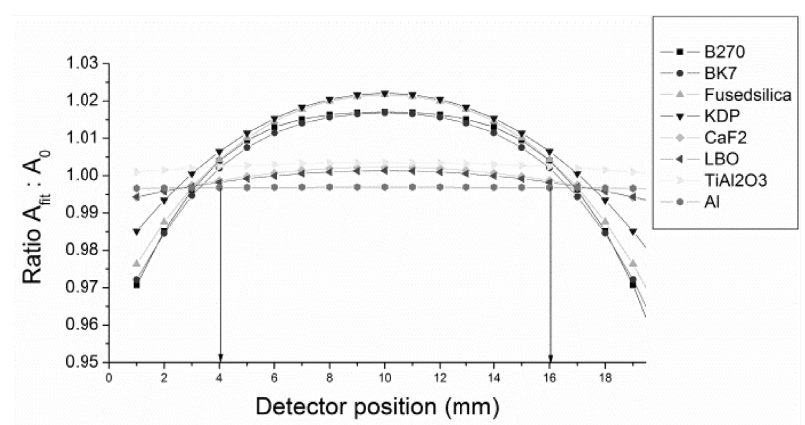

Fig.3.4. Evaluation of further heating scheme with exponential method: (a) two-face heating, (b) bulk heating.

\section{EXPERIMENTAL RESULTS}

\section{Comparison of simulated and experimental results for $4 \times 4 \times 20 \mathrm{~mm}^{3}$ crystals}

Because of the uncertainties concerning the exact sample parameters, it is reasonable to prove the FEM simulation results experimentally. For a direct comparison Fig.4.1 shows both the simulated (as seen in Fig.3.3) and experimental results (see Fig.4.1.b) for a front face heating scheme directly.

As a result of the large number of test samples, only a few detector positions were chosen for the measurement. The relative absorption of all shown test geometries is displayed in Fig. 4.2. The detector was placed at the lateral surface near the front face $(\mathrm{F})$ at all geometries. For the $8 \mathrm{~mm}$ and $20 \mathrm{~mm}$ thick samples the detector was placed at the lateral surface near the rear face (R) too. Only for the thick $20 \mathrm{~mm}$ samples another detector position in the middle (M, at $10 \mathrm{~mm}$ depth) was chosen. 
In Fig. 4.1.b the measurement results at different sample positions for the $4 \times 4 \times 20 \mathrm{~mm}^{3}$ sample geometry is shown together with all other apertures for $20 \mathrm{~mm}$ thick samples $\left(10 \times 10 \mathrm{~mm}^{2}\right.$ and $\left.20 \times 20 \mathrm{~mm}^{2}\right)$. Illustrated is the relative absorption ratio $A_{B 270} / A_{A l}$, where unity means identical measurement results, and therefore independence on the material properties. The interpolated experimentally determined 'optimized' detector position for the $4 \times 4 \times 20 \mathrm{~mm}^{3}$ sample geometry is around $6.5 \mathrm{~mm}$ (as seen in Fig.4.1.a). In comparison, the simulation considers the detector position around $7.5 \mathrm{~mm}$ as 'optimized' detector position. The results are looking similar, even though an exact confirmation with three different detector positions is difficult and the exponential fitting results depend on the selected intervals, which might induce some error of the simulation. Since the FEM simulation results agree with the experimental outcome, for further interpretations are used (as seen in Fig.3.4).

a)

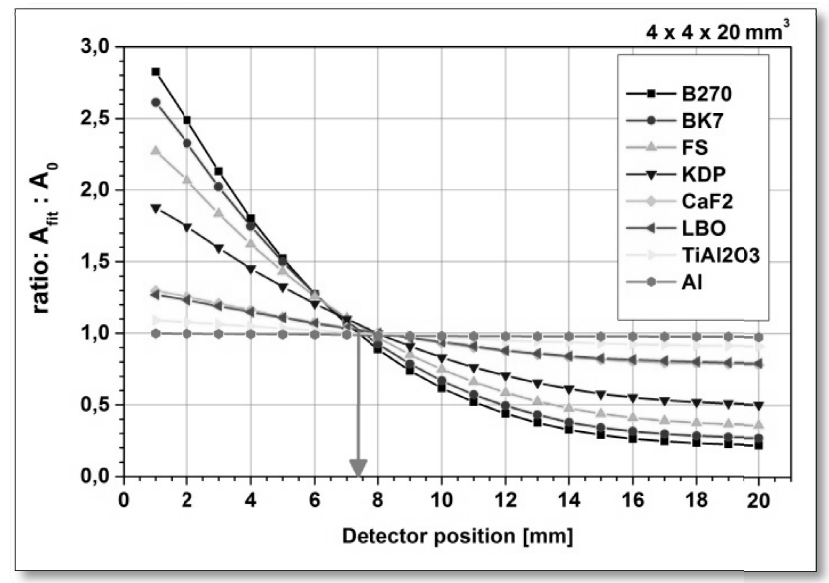

b)

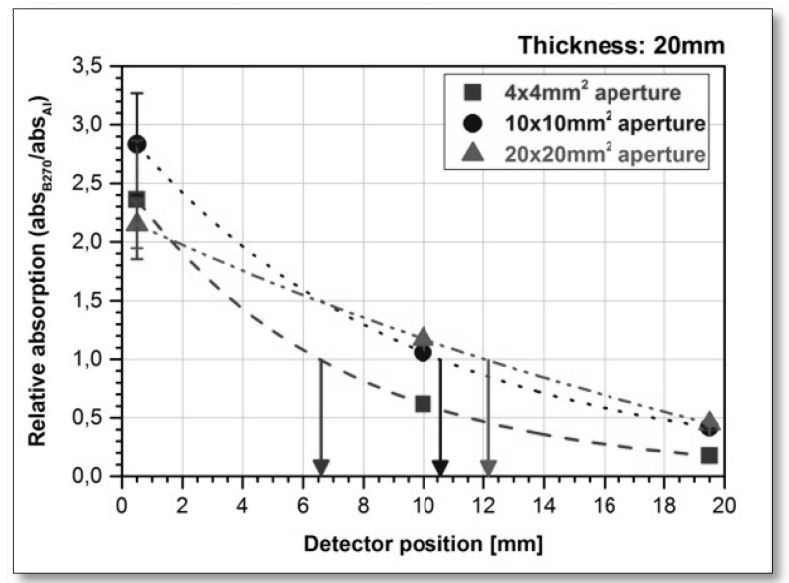

Fig.4.1. Evaluation of a suitable detector position for a $4 \times 4 \times 20 \mathrm{~mm}^{3}$ cuboid geometry with the exponential method: (a) simulated absorption for the $4 \times 4 \times 20 \mathrm{~mm}^{3}$ with varying thermal diffusivity (b) experimental absorption results for three different apertures $\left(4 \times 4 \mathrm{~mm}^{2}, 10 \times 10 \mathrm{~mm}^{2}\right.$ and $\left.20 \times 20 \mathrm{~mm}^{2}\right)$, which were fitted exponentially. The experimentally detected absorption values are scaled with the average absorption value of the respective blackened aluminum samples. The detector position precision is estimated to $\pm 0.5 \mathrm{~mm}$.

Another interesting point of the experimental absorption results is the shifting of the suitable detector position along the irradiation direction for higher aperture (for $10 \times 10 \mathrm{~mm}^{2}$ at $10.5 \mathrm{~mm}$ and for $20 \times 20 \mathrm{~mm}^{2}$ at $12 \mathrm{~mm}$ ). Near the front face Fig.4.2 shows high uncertainties of the relative absorption in particular for samples with high thickness $(20 \mathrm{~mm})$, which results from the inhomogeneous temperature curve for detector positions in particular closer at the irradiation center than the 'optimized' detector position (see sec. 5). 


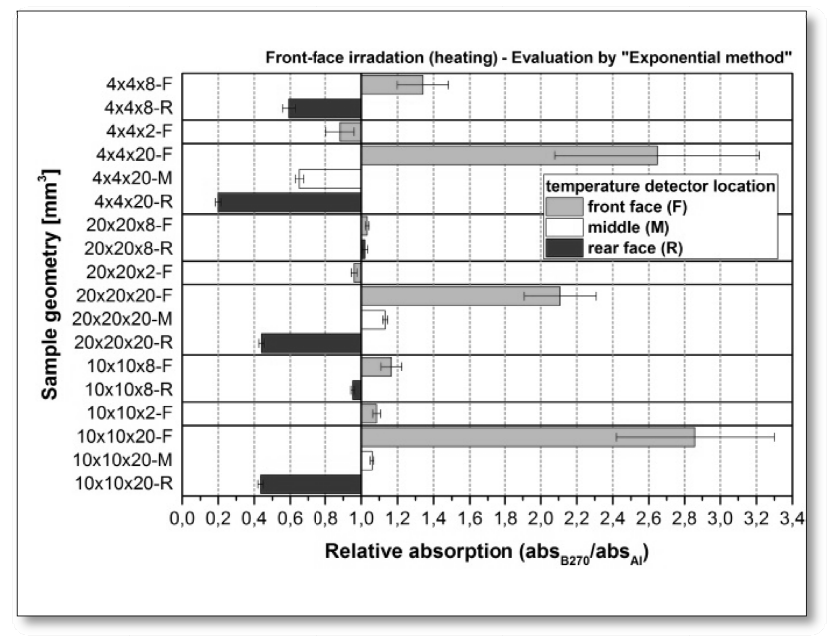

Fig.4.2. Experimental absorption results for the relative absorption quotient $A_{B 270} / A_{A l}$ of all test geometries evaluated with the exponential method with front face heating. All absorption values are scaled with the average absorption value of the respective blackened aluminum samples.

\section{EVALUATION WITH PULSE METHOD}

It has been demonstrated that in dependence on the heating scheme a suitable detector position can be found, at which the influence of heat conduction is compensated and the exponential method delivers correct results. However, the involved absorption profile of a sample, and therefore the heating scheme is usually not known. Consequently, an alternative evaluation method was tested as well. Obviously, this is not possible for the exponential method, because the temperature profile during the heating period is inhomogeneous in general. The pulse method, however, requires only the consideration of the cooling period of the measurement. Introducing a suitable delay after stopping irradiation of the sample gives a nearly homogeneous temperature distribution throughout the sample.

Fig.5.1.a shows a detected temperature curve exemplary near the front face of the sample geometry, which is evaluated with the pulse method for four different starting times of the cooling interval $t c 0$. This interval is varied exemplary and shows the change of the resulting absorptance $A$. This figure illustrates the general effect for low heat conduction when the detector position is closer to the irradiation center than the 'optimized' detector position. Because of an inhomogeneous temperature profile, the fits to the cooling curves for different starting times $t c 0$ lead to significant changes of the absorptance value $A$. The change of the absorptance and the fit deviation for different starting points of the interval $t c 0$ is shown in Fig.5.1.b. Shortly after stopping irradiation (for example $t c 0=267 \mathrm{~s}$ ), the temperature distribution is inhomogeneous, and too high absorptance values are observed for the fit $\left(A_{267 \mathrm{~s}}=182 \%\right)$ in conjunction with a high relative fit deviation. But for later instants of time (for example $t c 0=632 \mathrm{~s}$ and $t c 0=1050 \mathrm{~s}$ ), the sample temperature distribution gets homogenous and the evaluated absorptance values, as well as the very low relative fit deviation, are nearly constant (compare $A_{333 \mathrm{~s}}=95.2 \%$ and $A_{1050 \mathrm{~s}}=92.2 \%$ ).

The relative absorption of all shown test geometries, which were evaluated with the pulse method, is displayed in Fig. 5.2. All experimental results for the pulse method in Fig.5.2 show no influence of finite heat conduction for the apertures $10 \times 10 \mathrm{~mm}^{2}$ and $20 \times 20 \mathrm{~mm}^{2}$, especially when the temperature detector is placed near the heating center. This is possible for the pulse method, because the temperature data recorded during time periods of inhomogeneous temperature distribution can be excluded, in contrast to the exponential method.

For the $4 \times 4 \mathrm{~mm}^{2}$ aperture in general, the evaluated absorption is too low, especially near the rear face of the $4 \times 4 \times 20 \mathrm{~mm}^{3}$ sample geometry. Apparently, both tested evaluation methods could not measure a correct absorptance value at this detector position for this geometry.

In general, the pulse method showed higher uncertainties in the evaluation (a relative error of $2 \%-51 \%$ ) in contrast to the exponential method (a relative error of $1 \%-15 \%$ ). Without the $4 \times 4 \mathrm{~mm}^{2}$ aperture, the relative error changes for the exponential method to the relative error of $1 \%-15 \%$ and for the pulse method to the relative error of $2 \%-13 \%$, respectively. 
a)

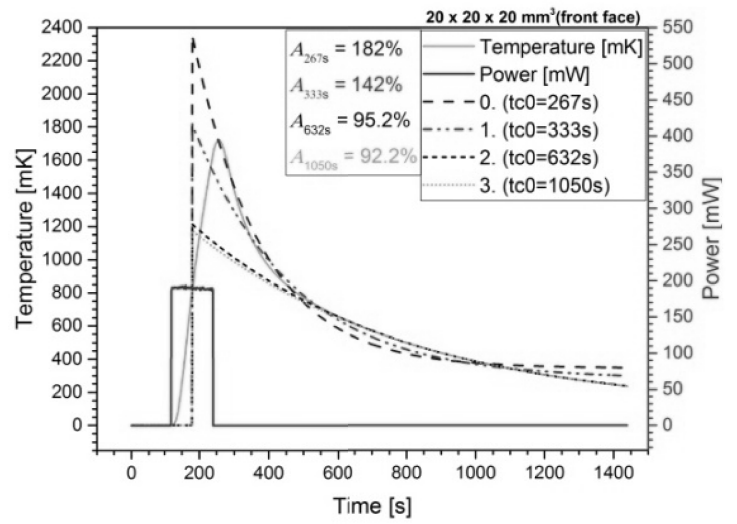

b)

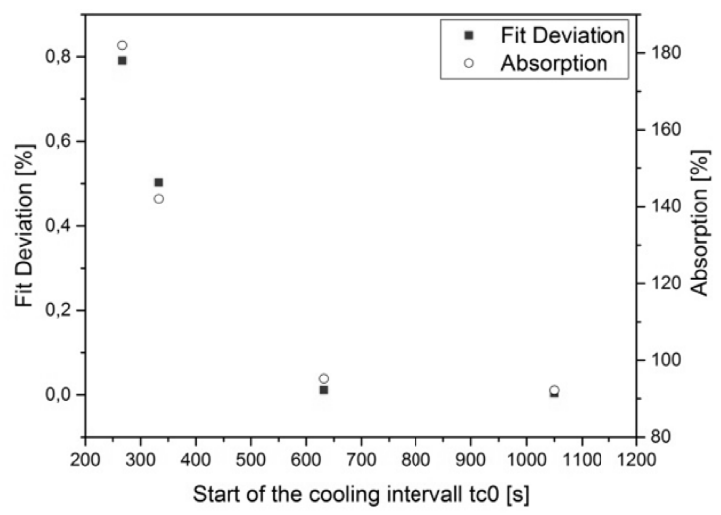

Fig. 5.1. (a) Exemplary detected temperature curve near the front face of the sample geometry. The absorptance values $A$ are evaluated with the pulse method for four different exemplary starting times of the cooling interval tc0. (b) The relative fit deviation as well as the resulting Absorption is shown for four different exemplary starting times of the cooling interval.

For the exponential method the absorptance values near the front face for sample geometries with high thickness (20 mm) should not be considered, because the method should mainly be used at the optimized detector position, at which the influence of heat conduction is compensated. Without these data points the relative error for exponential method is below $5 \%$, whereas the relative error for pulse method does not change $(2 \%-13 \%)$.

The $4 \times 4 \mathrm{~mm}^{2}$ aperture leads to the highest estimated errors and the corresponding uncertainties for the evaluation with the pulse method. This should be revisited in further experimental tests.

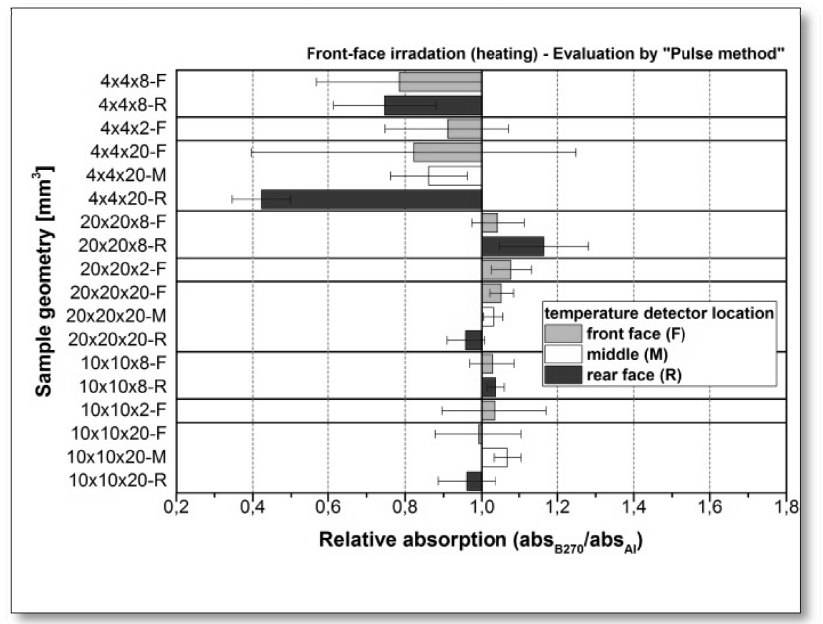

Fig.5.2. Experimental absorption results of front face heating for the relative absorption ratio $A_{B 270} / A_{A l}$ of all test geometries evaluated with the pulse method. All absorption values are scaled with the average absorption value of the respective blackened aluminum samples. 


\section{CONCLUSION}

By maintaining real measurement conditions, the influence of finite heat conductivity and the sample geometry on the measured absorption value were studied experimentally. This was achieved by comparing specific test samples of ${ }^{\circledR B} 270$ with aluminum samples which were modified to guarantee identical surface absorption. A suitable detector position, at which the measurement result is independent from the thermal diffusivity of the sample material, was determined experimentally for a front face heating scheme evaluated by the exponential method. The experimental results confirm the simulations by FEM so that the simulations can be used for further studies, like two-face or bulk heating.

Furthermore, the experimental results indicate the pulse method as a possible alternative evaluation method (if a suitable detector position cannot be identified). However, the measurement results for small test samples showed high uncertainties for the pulse method, which should be analyzed in further studies.

\section{REFERENCES}

[1] ISO 11551, "Test method for absorptance of optical laser components, “ International Standard, International Organisation for Standardisation, Geneva Switzerland (2003)

[2] Balasa, I., Jensen, L. O., and Ristau, D., "Laser calorimetric absorptance testing of samples with varying geometry," OPTICAL ENGINEERING 53(12), 122503 (2014)

[3] Van Heerden, S.P., Klopper, W., Prinsloo, F.J. and Forbes, A., “Influence of finite absorption in transmission optics on the propagation of high average-power pulsed $\mathrm{CO}_{2}$ laser beams," Proc. SPIE 2870(6), 515-520 (1996).

[4] Zimmermann, P., Ristau, D. and Welsch, E:, "Potentiality of the Photothermal Surface-Displacement Technique for Precisely Performed Absorption Measurement of Optical Coatings," Appl. Phys. A 58(4), 377-383 (1994).

[5] Welsch, E. and Ristau, D., " Photothermal measurements on optical thin films," Appl. Opt. 34 (31), 7239 -7253 (1995).

[6] Li, B., Blaschke, H. and Ristau, D., " Pulsed photothermal deflection with top-hat beam excitation,” J. Appl. Phys. 100 (5), 053509 (2006).

[7] Mühlig, C., Triebel, W., Kufert, S. and Bublitz, S.," Characterization of low losses in optical thin films and materials," Appl. Opt. 42, C135 (2007).

[8] Mühlig, C., Bublitz, S. and Paa, W.," Laser induced deflection (LID) method for absolute absorption measurements of optical materials and thin films," Proc.SPIE 8082(12), (2011).

[9] Alexandrovski, A., Fejer, M., Markosian, A. and Route, R.," Photothermal common-path interferometry (PCI): new developments," Proc. SPIE 7193, 71930D (2009)

[10] Van Dijk, M.A., Lippitz, M., Stolwijk, D. and Orrit, M.," A common-path interferometer for time-resolved and shotnoise-limited detection of single nanoparticles." Opt. Express 15, 2273-2287 (2007).

[11]Li, B., Martin, S. and Welsch, E.," Pulsed top-hat beam thermal-lens measurement for ultraviolet dielectric coatings," Opt. Lett. 24(20), 1398 (1999).

[12] Wu, Z., Kuo, P.K., Lu, S. and Gu, S.T.," Laser-induced surface thermal lensing for thin film characterizations," Proc. SPIE 2714(11), (1996).

[13] Reichling, M., Welsch, E. and Matthias, E.," Thin-film characterization and photothermal absolute calibration measurements using high-frequency electric currents," Proc. SPIE 1781(9), (1993).

[14] Welsch, E., Walther, H.G., Eckardt, P. and Lan, T.," Low-absorption measurement of optical thin films using the photothermal surface-deformation technique," Rev. can. phys. 66(7), 638-644 (1988).

[15]Li, B., Blaschke, H. and Ristau, D.," Combined laser calorimetry and photothermal technique for absorption measurement of optical coatings," Appl. Opt. 45, 5827-5831 (2006).

[16] Willamowski, U., "Standardisierbare Verfahren zur präzisen Charakterisierung des Absorptions-, Reflexions- und Transmissionsgrads optischer Komponenten," Dissertation, Gottfried Wilhelm Leibniz Universität Hannover, Hannover/Germany (1998).

[17] @ 2017 by COMSOL Inc., http://www.comsol.com/comsol-multiphysics

[18] Willamowski, U., Ristau, D., Welsch, E.,“ Measuring the absolute absorptance of optical laser components,” Appl. Opt. 37(36), 8362-8370 (1998). 